REYISTA

dete

CEPA

NUMERO 62

AGOSTO 1997

SANTIAGO DE CHILE

OSCAR ALTIMIR

Direcsor

EUGENIO LAHERA

Secretario Técnico

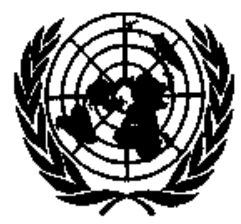

NACIONES UNIDAS 
Estado, comunidad y sociedad en el desarrollo social

Fermando Henrique Cardoso

Un balance de las reformas estructurales neoliberales en América Latina

Joseph Ramos

Deuda y sostenibilidad ffscal: ise repite la historia?

Guillermo E. Perry

Reformas petroleras: las opciones en juego

Fernando Sánchez Albavera

Las organizaciones indígenas: actores emergentes en

América Latina

Rodolfo Stavenhagen

El empleo rural no agropecuario en el Istmo Centroamericano

Jürgen Weller

Marginalidad e integración social en Uruguay

Rubén Kaztman

La política comercial en el marco de la Organización Mundial

de Comercio

Diana Tussie

Comercio y medio ambiente: ¿luz verde o luz roja?

Helga Hoffmann

Anclas nominales y escenarios de coordinación macroeconómica en el MERCoSUR

Gonzalo Rodríguez Prada

Políticas de promoción de exportaciones en Centroamérica

Larry Willmore

Publicaciones recientes de la CEPAL 


\section{Estado, comunidad y sociedad en el desarrollo social}

\section{Fernando Henrique Cardoso}

Presidente de la Repúbica

Federativa del Brasil

La Cumbre Mundial sobre Desarrollo Social realizada en Copenhague el 1 I y 12 de marzo de 1995, puso una vez más sobre el tapete los ideales que dieron origen a las Naciones Unidas en la Conferencia de San Francisco, y que se han reafirmado en muchos foros de la organización. El mantenimiento de la paz y la seguridad, sin duda dimensiones insustituibles de la convivencia internacional, no fueron los únicos objetivos de la Conferencia de San Francisco; allí se buscó también cimentar una convivencia que hiciera posible un desarrollo más armonioso. La Carta de las Naciones Unidas que emanó de esa reunión fue expresión evidente de un espíritu humanista, y de la búsqueda de ideales democráticos y de valores que centren en el ser humano la preocupación de los gobernantes.

En Copenhague quedó en claro que el problema social y la búsqueda de un desarrollo que respete el medio ambiente, sea democrático y conduzca a una mayor equidad no son preocupaciones exclusivas de los países subdesarrollados o en desarrollo, sino que también se dan en los países desarrollados. Tal vez se haya vuelto a tomar conciencia de que no se trata sólo de una dualidad, como se decía en otra época, sino de algo inherente al corazón mismo del estilo de desarrollo de tas sociedades contemporáneas.

[.] Texto traducido y revisado de la intervención del Presidente Cardoso en la Primera Conferencia Regional de Seguimiento de la Cumbre Mundjal sobre Desarrotlo Social (São Paulo, 6 al 9 de abril de 1997).
Por lo tanto, una vez más es importante dar continuidad al impulso reflexivo y de acción generado en Copenhague. Y la CEPAL, por su gran experiencia en América Latina y el Caribe, está capacitada para ayudar a los países de la región a repensar estos temas con renovada creatividad.

El compartir experiencias -aunque muchos de los intentos realizados en la región se hayan frustrado- es lo que puede ayudarnos a reorientar las políticas de desarrollo en una dirección consecuente con nuestros ideales, que siguen -y han de seguir siendo- los proclamados desde 1945: ideales de un desarrollo con mayor equidad, inspirado en valores democráticos y humanistas.

Los diez compromisos asumidos en Copenhague surgieron en un momento oportuno y a la vez complejo.

Oportuno, porque, después de mucho tiempo en que casi sin darnos cuenta se había vuelto a pensar en el desarrollo sólo en términos de desarrollo económi$\mathrm{co}$, de desarrollo de las fuerzas de mercado, en la Cumbre de Copenhague se volvió a hablar de la relación entre lo económico y lo social.

Sería casi patético imaginar que, en el mismo momento en que se producía la caída del Muro de Berlín, en que las experiencias de socialismo real tenían el desenlace que tuvieron, de pronto todos comenzaran a apostar por una especie de economicismo desbocado y que se comenzara a creer que el mercado era el camino para la redención de la humanidad. 
En Copenhague se nos señaló otra vez la necesidad de retomar temas como la erradicación de la pobreza, el pleno empleo, la integración social, el respeto a la dignidad del ser humano. $Y$ de poner fín a la ilusión economicista, de reconocer una vez más que en un proceso social, en un proceso histórico, los valores son fundamentales.

Cuando digo que los valores son fundamentales, no pretendo cambiar la ilusión economicista por un idealismo simplista, imaginando que basta con los valores. Evidentemente no es asf. No basta con los valores, no basta con las buenas intenciones. En realidad, contar con una base económica estable, con una moneda estable, son condiciones esenciales para el desarrollo.

Por lo tanto, no se trata de cambiar un criterio simplista por otro - igualmente simplista pero diametralmente opuesto-, según el cual la voluntad política organizada podría sustituir a las condiciones materiales de producción o a los límites impuestos por la base física y la modalidad de organización de la producción.

Con este reparo, no cabe duda de que ha llegado el momento de volver a ocuparse de temas que, por lo demás, siempre han sido los temas de la CEPAL, de quienes concebían un desarrollo que ofreciera un mayor bienestar a la mayoría de la población.

Pero aunque sea oportuno, también es complejo plantear otra vez con gran vehemencia el tema del desarrollo y el desafío de alcanzar la igualdad, es decir el desafío de lo social. Y lo es porque refleja una suerte de paradoja.

Tal como sería paradójico sustituir un modelo socialista - que se deformó mucho- por una idea simplemente de mercado, también sería paradójico que precisamente cuando volvemos a hablar de lo social, tengamos que pedirle al Estado que aplique un conjunto de políticas y tome una serie de medidas, cuando en cierto sentido ese Estado no es ni eficaz ni eficiente.

Pero cuando digo que le estamos exigiendo más al Estado --porque la voluntad política se organiza y porque las políticas públicas son las que permiten en cierta medida corregir los desequilibrios creados por el mercado- en un momento en que el Estado tiene limitaciones, lo que digo es que no quiero resignarme a las limitaciones que le impiden actuar con eficacia.

Es cierto que tiene limitaciones. En primer lugar, y con eso no digo nada nuevo, ahora mismo vemos que la capacidad reguladora de los Estados nacionales se ha reducido enormemente. Para demostrarlo basta con referirse a las corrientes de capital que hoy en día in- quietan incluso a los que normalmente descansan de noche, es decir a los presidentes de los bancos centrales. Ni siqujera ellos están tranquilos, porque no logran controlar esas corrientes de capital. Ya no se puede hacer frente a ese desafío desde la estera de competencia exclusiva del aparato estatal.

Pero no se trata sólo de las corrientes internacionales de capital; el sistema de producción mundial también se reestructuró, y se ha reorganizado de tal manera que la producción está interconectada a nivel mundial, to que también contribuye a reducir la capacidad de los Estados nacionales para responder a algunos de los desafíos que se plantean.

$Y$ no hay muchas alternativas a esta interdependencia. La revolución no sólo tecnológica sino también organizacional plantea un nuevo desafío a quienes consideramos que se necesita un conjunto de políticas públicas que permitan un mayor equilibrio en el proceso de desarrollo.

Esto significa que se espera de la voluntad política organizada del Estado, de la sociedad, un conjunto de medidas que, por otro lado, se ven limitadas por las propias realidades contemporáneas, que quitan capacidad de acción al Estado en áreas que en el pasado eran inherentes a la noción de Estado soberano y de Estado nacional.

Con esto no se pretende destacar lo paradójico o provocar perplejidad, reacción psicológica que no tiene ninguna importancia teórica ni práctica. En realidad, de lo que se trata es de encontrar mecanismos que permitan reformar las estructuras públicas para que estén a la altura del desafío que se plantea.

La justicia social y el desarrollo social son imposibles si nos conformamos con el debilitamiento del Estado, tanto por las razones mencionadas como por la incapacidad, en muchos casos burocrática, de adoptar decisiones para responder al aumento de las demandas sociales, estrechamente vinculado a la democratización.

La democratización y el aumento de las demandas sociales se hacen sentir en forma más acelerada y con más fuerza precisamente en los países que han experimentado una apertura democrática y que exhiben mayores desigualdades, y donde, por lo tanto, el Estado también está siendo puesto en jaque por fuerzas externas e internas.

Surge entonces el desafío de reconstruir las formas de organizar la acción política y, sobre todo, de redetinir los brganos que se ocuparán de las políticas públicas. Al redetinin hay que escoger qué hacer y qué no hacer. Esa es la situación actual: hay que definir lo 
que le corresponde hacer al gobierno, lo que le es posible hacer, y la forma de rehacer los mecanismos gubernamentales para que ellos puedan actuar.

Hay que insistir en este tema, a fin de que el Estado recupere la fuerza necesaria para canalizar los deseos de la sociedad y responder a los anhelos de bienestar social. En otras palabras, hay que "desprivatizar" el Estado.

Paradójicamente, muchas de las fuerzas que podrían contribuir a esa desprivatización endiosan al Estado en su forma actual - como si éste no fuese producto de la etapa anterior, en la que hubo una estrecha vinculación entre los sectores privados y el Estado-y terminan impidiendo la transformación que le permitiría democratizarse y convertirse en un instrumento eficaz para difundir ampliamente el bienestar social y para dar a la población un mayor acceso a todo lo que necesita para su integración social.

En nuestra región nunca hubo un Estado de bienestar social. Lo que predomina en nuestra región es lo que podríamos llamar un Estado de "malestar social". Es un Estado omnipresente, contaminado por los intereses privados, buenos o malos; y además, paralizado por las fuerzas corporativas que surgen de su interior, por la burocracia. Con frecuencia el Estado y la sociedad civil, con lo que ambos tienen de perverso, coinciden en la corrupción.

Un Estado así tiene que ser objeto de reformas valientes, profundas, para que se transforme democráticamente en uno capaz de acoger los anhelos de los sectores marginalizados, excluidos, y de los sectores que, si bien están integrados en la sociedad, requieren una distribución más justa del ingreso.

Por eso, en nuestra región no es necesario analizar el tema de la crisis del Estado de bienestar, ya que nunca liegamos a tenerlo. Lo que sí se ha dado es una crisis del Estado de "malestar social". Al reconstruirlo habría que evitar los errores que llevaron a la crisis del Estado de bienestar cuando éste, por un conjunto de circunstancias, dejó de generar el bienestar que ofrecía.

Beneficiándonos de la experiencia histórica, es posible concebir reformas del Estado y una forma de actuación de ese mismo Estado que permita superar la paradoja que se señaló más atrás. Abandonemos la ilusión de que el mercado por sí solo va a traer igualdad; lo que queremos es más y más igualdad, no con la perpetuación de un Estado de "malestar social" y privatizado, sino a través de una reforma del aparato estatal que permita transformarlo en un instrumento de progreso social.
Esto lleva a replantear temas que siempre fueron fundamentales, pero que ahora van adquiriendo cada vez más importancia. No me refiero solamente al tema de la reforma del Estado. La educación se ha convertido en un elemento esencial de todo el proceso, porque ante todos los cambios que ha sufrido el sistema de producción y ante todos los desafíos que tendremos que encarar, o bien los ciudadanos tienen capacidad de adaptación y las condiciones en que se da dicha adaptación se van perfeccionando cada vez más, o bien hablar de inclusión social es una hipocresía. No habrá inclusión social para los que no estén preparados.

La educación tendrá un sentido mucho más amplio, porque no se limitará a la alfabetización, ni siquiera a una capacitación formal, sino que supondrá la incorporación efectiva en la vida cotidiana de los ciudadanos, de técnicas que les permitan informarse y tomar decisiones, porque sin información no se puede optar y se es víctima de la manipulación. La capacidad de adaptación es necesaria incluso para buscar una ocupación, si no un empleo.

Como todos sabemos, el actual proceso de globalización puede agravar severamente la exclusión social. No estoy diciendo que debamos darle la espalda al proceso de globalización, porque no hay otra alternativa. ¿Qué alternativa podría haber? ¿La de la autarquía? ¿Dónde? ¿Cómo? La producción está cada vez más dispersa y es cada vez más competitiva y dependiente de la tecnología, la mayor parte de la cual no está en manos de un solo sector. No hay alternativa a la globalización y eso es un hecho innegable. Lo que cabe entonces es buscar qué se puede hacer, dadas las circunstancias, para que la exclusión no limite las posibilidades de que los temas que nos ocupan salgan del ámbito teórico para convertirse en mecanismos de transformación.

Evidentemente, la educación no es el único medio para hacer frente al desatio. La competitividad es un requisito de la inclusión, una norma a la que tampoco podemos escapar. $Y$ todo esto tiene consecuencias, bien conocidas, en lo que respecta a la oferta de empleo. De hecho, habría que modificar el concepto mismo de empleo.

Cuando se observan los cambios que se han producido en la estructura del empleo y los efectos que ellos han tenido sobre la ocupación en Europa, Estados Unidos y Japón, se ve claramente que la globalización no se traduce automáticamente en una deterninada tasa de desempleo. La tasa varía y depende de la intervención del sector público, de las condiciones sociales, de las normas institucionales y jurídi- 
cas que regulan las relaciones laborales; de la capacidad cultural, valorativa, de comprender que en una sociedad como la que se está delíneando la movilidad, incluso la geográfica, pasa a ser un requisito de la adaptación. Se producirá un acelerado desplazamiento de sectores productivos de una region a otra, como el que se está dando en Brasil y del cual São Paulo es un ejemplo, $y$ este movimiento seguramente beneficiará al nordeste del país. Pero no beneficiará al trabajador que perderá su empleo en un lugar dado si no somos capaces de crear nuevos empleos en ese lugar, o de trasladar al trabajador del sector secundario al terciario, o de darle, como en Estados Unidos, una gran movilidad geográfica que haga posible la búsqueda de empleo. Esa movilidad es el polo opuesto de nuestros valores, que dan preferencia a la estabilidad en un sentido amplio, al no desplazamiento del individuo y la familia de un lugar a otro.

Todo esto exige una nueva perspectiva cultural. Por eso he hecho hincapié en la educación, que es un instrumento de socialización, de nuevos valores, de desafíos, y de preparación y motivación para hacer frente a esos desafíos.

La situación no puede seguirse planteando en términos de disyuntiva: primero lo económico y después lo social. $O$ afirmando que to social es lo realmente importante, porque el ser humano está por encima de todo lo demás. Tampoco con una combinación indiscriminada de ambas posibilidades, que no tiene sentido. Hay que prestar atención a lo económico y a lo social al mismo tiempo, y aún más, hay que precisar qué significa exactamente esta expresión, ya que de hecho a veces no se da todo al mismo tiempo, sino primero o bien lo social o bien lo económico.

Y no hay que tener una visión estática. A veces to social viene primero porque, en determinadas circunstancias, lo que impulsa el proceso de transformación es la educación, son los valores. En otras circunstancias, el impulso lo da un desarrollo tecnológico que proviene del exterior, producto de la educación y valores foráneos, que de todos modos influyen en nuestra región.

Por eso, hay que tener una visión casi caleidoscópica, no guiarse por reglas fijas sobre el orden de precedencia, $y$ preocuparse constantemente por establecer vinculaciones entre uno y otro aspecto. Si un proceso se desencadena en el plano económico, hay que vincularto a lo social. Si se desencadena en el plano social, hay que vincularlo a lo económico; de lo contrario, no tendrá base sólida ni continuidad.
Por consiguiente, es preciso poner límites al raciocinio extremadamente mecánico, en términos de disyuntivas, y aceptar el reto de reflexionar a partir de situaciones concretas.

Refiriéndome ahora al caso de Brasil, diría que el esfuerzo que hemos hecho para estabilizar la economía y para consolidar y afianzar la democracia, así como nuestro interés por el desarrollo social, responden a las consideraciones mencionadas. Se inspiran en ellas, aunque no siempre logran los resultados deseados.

Indudablemente, cuando formulamos el plan de estabilización conocido como Plan Real rechazamos la recesión como mecanismo de estabilización. En realidad, desde 1993 a 1997 inclusive la economía brasileña habrá crecido en un 25\%, en caso de que en 1997 el crecimiento sea de 4 a $5 \%$. En una economía que hoy en día representa 700000 millones de reales, un $25 \%$ es un incremento alto, que además se ha alcanzado en el mismo período en que estaba vigente el plan de estabilización.

Desde que se comenzó a aplicar el Plan Real, a partir de 1993, la remuneración media aumentó en un $42 \%$, aunque de manera discontinua. Más aún, su incremento fue mayor en el sector informal que en el formal. E incluso aumentó más en el caso de los trabajadores independientes y por cuenta propia que en las demás categorías. Aunque hubo deficiencias, la verdad es que fue posible — por lo menos, ha sido posible hasta ahora-conciliar en este plano ta estabilización de la moneda con el crecimiento económico y la distribución del ingreso.

Por primera vez, las estadísticas sobre distribución del ingreso, que como es sabido difícilmente acusan cambios, dieron señales de que quienes más ganan ganarán más, pero en proporción más baja que los que ganan menos, aunque éstos sigan ganando muy poco. Assí, hubo indicios de una modificación de la distribución funcional del ingreso, lo que es muy importante y debe mantenerse, aunque no es fácil hacerlo.

Las estadísticas también muestran que, en las seis áreas metropolitanas más grandes, 13 millones de brasileños superaron el umbral de la pobreza entre 1993 y 1995 , lo que es un buen ejemplo de los efectos sociales que puede tener un plan de desarrollo económico. Sin partir de una idea fija sobre to que viene primero y lo que viene después, se pudo hacer un esfuerzo en esa dirección, dentro de las limitaciones existentes.

Respecto al empleo no puedo ser tan categórico, porque los procesos de transformación global suelen plantear desafíos a las economías y, en ciertos perío- 
dos y en algunas regiones, el índice de desempleo aumenta. En promedio - por lo menos usando el indicador que nos permite hacer comparaciones internacionales, el del Instituto Brasileño de Geografía y Estadística (IBGE) - la tasa de desempleo en Brasil fluctuó entre el 5 y el $5.5 \%$ en los años indicados. Con otros tipos de indicadores las cifras pueden resultar más altas. Pero todos concuerdan con respecto a la tendencia, creciente o decreciente. Cada cual a su nivel, porque los resultados dependen de cómo se formule la pregunta (si el trabajador está buscando empleo desde hace una semana, un mes, tres meses; qué edad tiene el encuestado, etcétera). Pero si se aplica de manera homogénea la misma técnica lo que importa es la fluctuación. Y desde que se empezó a aplicar el Plan Real la fluctuación registrada ha sido mínima, cualquiera sea la metodología que se aplique.

Ha habido recuperación, que es notoria aunque no permita afirmar que se producirá una absorción de desempleo. Evidentemente, no se puede garantizar la continuidad del proceso si no se afianza la reforma del Estado, asunto dificilísimo porque provoca una fuerte oposición, con distintos pretextos, de la izquierda y la derecha.

La reforma del Estado perjudica a ciertos intereses y todo to que perjudica a ciertos intereses despierta oposicion. Por eso hay que seguir luchando para avanzar en este proceso. Pero la reforma no supone sólo una lucha contra el corporativismo de los funcionarios, sino que implica mucho más.

Sobre todo, implica rehacer en las áreas sociales los mecanismos a través de los cuales se vinculan el aparato estatal y la sociedad civil. Hay muchos ejemplos concretos. El Ministerio de Educación, que ha tomado muchas medidas con ese objeto, ha dispuesto entre otras cosas que parte de los recursos para las escuelas se les entreguen directamente, sin pasar por otras instancias, políticas en muchos casos, burocráticas en otros. En el área de la salud, donde el mayor obstáculo es la búsqueda de mecanismos que establezcan un vínculo directo entre las demandas de la sociedad y el aparato estatal, también hay problemas políticos, pero éstos se plantean a nivel de la política sustantiva, no de la mediación, de la transferencia de recursos.

Por otra parte, hoy en día el Estado, sobre todo tratándose de una federación como la brasileña, no puede seguir siendo de carácter burocrático unitario. Tiene que haber una descentralización, que ya está en marcha en el sector de la educación, de la salud, y que esperamos se vaya consolidando en la reforma agra- ria, sin lo cual no se darán las condiciones necesarias para que progrese un país del tamaño de Brasil.

Naturalmente, el gobierno central debe tener, en primer lugar, una responsabilidad financiera, tal vez la principal. En segundo lugar, debe tener la capacidad de definir políticas. En tercer lugar, to que es muy importante y poco común en la región, la capacidad de evaluar los efectos de las políticas, mediante un control de sus resultados. En cuarto lugar, lo que es fundamental para ejercer ese control, debe tener vinculaciones con la sociedad, los sindicatos y los partidos de oposición.

Esto es lo que ocurre actualmente en la educación y la salud. Y en vivienda, por ejemplo, no se hace nada sin pasar antes por un mecanismo que no sólo está subordinado al poder político legítimo, sino también a un diálogo con la sociedad. En ese diálogo se amplía el espectro y en el proceso de adopción de decisiones se incluye también a sectores minoritarios o que son adversarios del gobierno central. Y el gobierno central nunca pone obstáculos de carácter partidista a la distribución de recursos, porque reconoce la importancia de que éstos lleguen adonde se los necesita y de que haya un mecanismo transparente de control de su transferencia.

Por lo tanto, la transformación del aparato estatal no es una simple "desprivatización" del Estado, en el sentido de independizarlo de los intereses privados enquistados en él. También supone modificar la relación burocrática. No se trata sólo de que el Estado se margine de ciertas áreas de la actividad económica en las que existan recursos de capital, para concentrarse en otras, sino de que haya transformaciones fundamentales en el ethos de la administración pública, sin lo cual no puede haber desarrollo con más equidad, porque las fuerzas del mercado por sí solas no son capaces de generar esa equidad. El sistema de gobierno tiene que ser abierto, democrático, y hacer participar a Jos sectores de oposición, lo quieran o no, en el proceso de toma de decisiones (naturalmente, sobre la distribución de los recursos, no en lo que respecta a la orientación general del proceso, que en un régimen democrático es decidida por la mayoría). Esto exige una amplia transformación: la "desprivatización" del Estado, su desburocratización, su descentralización, son condiciones sine qua non para poder avanzar.

Por supuesto, hay ciertas situaciones en nuestros países —en Brasil, país inmenso y tan lleno de desigualdades- que claman por medidas más enérgicas. Una de ellas está vinculada con el acceso a la tierra. Tradicionalmente, por su evolución económica e his- 
tórica, Brasil ha sido un país de latifundios. El latifundio ha sobrevivido con mucha fuerza, y luego de grandes cambios en el sector agrícola, hoy convive con el sector productivo empresarial y sigue estando presente en la vida brasileña.

Entretanto, amplios sectores de la población viven en la pobreza: hay una gran pobreza rural y urbana y, cada vez más, una combinación de ambas. Y cada vez más, simbólicamente, pedir el acceso a la tierra es el medio indirecto de pedir equidad. Pero pese a que se puede y se debe reconocer este proceso, no hemos hecho una reflexión profunda sobre las consecuencias del acceso a la tierra ni sobre las posibilidades, los costos y la índole de la reforma agraria que se pueda realizar.

En cuanto al ritmo de asentamiento, el promedio de Brasil en el pasado era ridículo: diez mil familias al año. En este gobierno se han asentado poco más de cien mil en dos años y este año se proyecta asentar a otras ochenta mil. En comparación con lo que se necesita no es nada; en comparación con lo hecho en el pasado es bastante, y en comparación con los recursos es mucho. Cada asentamiento cuesta de partida 25 mil reales. Y lo que ha ocurrido en Brasil es que, una vez asentadas, las familias no se "emancipan" sino que siguen siendo objeto de la acción estatal; por lo tanto, el flujo de recursos continúa, lo que debe tomarse en cuenta.

La sociedad tiene que decidir si desea o no, si puede o no, llevar adelante este proceso. Y si decide hacerlo debe dar los medios - pagar impuestos-para que la decisión se transforme en una realización concreta. Para asentar un millón de familias, se necesitarían 25 mil millones de reales, para comenzar.

$\mathrm{Y}$ tras el asentamiento, hay que prestar atención a la unidad familiar de producción. Un programa establecido recientemente -el PRONAF- ha destinado alrededor de 600 miliones de dólares a dar apoyo a la pequeña unidad familiar.

En Brasil, en muchos casos, los recursos están disponibles. Hay voluntad política en la cúpula, pero no existen los mecanismos necesarios para que esos recursos fluyan en forma adecuada y lleguen a manos de quienes los necesitan. Las instituciones del Estado -un Estado creado para provocar "malestar social", para la gran empresa, para las grandes corporaciones, para los contratistas, para los bancos- no tienen canales para llegar al pueblo. Está empezando a delinearse entonces una nueva situación en que, gracias a la estabilidad y a la reanudación del crecimiento, se comienza a disponer de recursos, pero faltan los medios para utilizarlos adecuadamente, dando prioridad a los sectores más pobres de la población.

Ese problema es de gestión y a la vez político, porque en muchos casos lo que obstaculiza la gestión es el clientelismo, son los intereses locales. Y hasta en las mismas estructuras burocráticas suele haber trabas, por razones políticas, por incompetencia, o por diferencias ideológicas, que impiden que los recursos fluyan.

Sin una reforma del Estado no puede haber política social, y sin una mayor coordinación y capilaridad con la sociedad, la política social se marchita en la garganta de los que gritan y en la pluma de los que otorgan pero saben que su aporte no tiene efectos prácticos, porque no hay capacidad social para llevar a la práctica las decisiones.

Este tema está íntimamente relacionado con el de la equidad, que es esencial, como el tema de la tierra. Hay numerosos interrogantes y son muchos los que ofrecen respuestas o recetas con gran soltura. Pero cuando se reflexiona y se profundiza, se hace evidente que es necesario trabajar todos juntos, que nada se resuelve simplemente con gestos de voluntad ni con actos de protesta, y que es preciso reorganizar las finanzas para que el Estado pueda funcionar e interactuar con la sociedad civil.

Otro tema vinculado a la equidad que deseo destacar —en el espíritu de Copenhague, con una visión no economicista de los cambios- es el de los derechos humanos, fundamental en un país como Brasil. En él se está creando la Secretaría Nacional de Derechos Humanos, porque la preocupación por los derechos humanos no puede limitarse a gestos de buena voluntad ni de los gobernantes ni de quienes son sensibles a este tema. Tiene que haber un proceso de reeducación que abarque el ámbito de los valores, así como continuidad en la acción y una insistencia constante en el tema.

En sociedades excluyentes como la nuestra, que se han habituado a una desigualdad tan marcada, la violencia aparece como un subproducto. Y pronto deja de ser producto de la pobreza y se arraiga en una especie de tolerancia de lo intolerable, en una aceptación de prácticas abyectas contra las cuales, en último término, nos corresponde a los gobernantes protestar. Pero protestar ¿contra qué? ¿Y quiénes son los responsables? Hay responsables: todos nosotros. Este es un proceso amplio que exige continuidad en la acción.

Para que el proceso de reeducación tenga continuidad, se necesita una especie de faro que ilumine, 
que actúe como la buena conciencia del país y señale constantemente los errores, aun a sabiendas de que en muchos casos esos errores no pueden corregirse en un momento dado, y aun a sabiendas de que hay culpables y de que no basta con culparlos, porque hay que ir mucho más allá: hay que desarrollar otro tipo de valores, otro tipo de solidaridad.

Quienes han estudiado sociología conocen a Fernando Tönnies y una expresión clásica de la sociología, que es la distinción entre las palabras alemanas Gemeinschaft y Gesellschaft, comunidad y sociedad. Con la expresión Gemeinschaft los autores de esa corriente, hace casi un siglo, se referían a las relaciones cara a cara, directas, propias de una comunidad, a la posibilidad de una experiencia común: las personas se unen porque tienen una vivencia en común. En una situación de Gesellschaft, que es societal, hay una norma contractual y no se necesita la solidaridad que nace de una vivencia en común. Una y otra expresión eran vistas en términos de oposición.

Ahora bien, en el mundo de hoy, con los cambios que se han producido, con los medios de comunicación actuales, con lo instantáneo de los procesos, de algún modo las sociedades comienzan a tener vivencias en común: de violencia, de bienestar, de miedo, de desafío económico, de logros materiales. Vuelve a surgir la posibilidad de que se dé esa situación incluso en la relación societal.

Nuestro desafío es cómo superar tanto la $G e$ meinschaft, la comunidad, como la Gesellschaft, la sociedad, la relación societal: cómo superarlas juntándolas, en el sentido específico de la noción de superación dialéctica.

Debemos superar esa oposición entre lo económico y lo social y lo político, entre lo público y lo privado. Ese es el desafío. Una visión nueva del mundo exige la búsqueda de un concepto que nos lleve a reestructurar nuestro pensamiento, dejando atrás esa oposición entre comunidad y sociedad que nos fascinó a todos hace tantos años, y a hacernos sentir capaces de una acción congruente con los desafíos de hoy.

Esa acción ha de basarse en un gran impulso tecnológico, que permita estar presente en todos los ámbitos. Y esa acción sólo se realizará, y la distinción entre sociedad y comunidad sólo se superará, si volvemos al tema de la ética, de los valores, no como algo amenazante, no como un discurso moral vacío, sino como un modo eficaz de motivar una acción transformadora.

(Traducido del portugués) 